\title{
Scaling behavior of randomly alternating surface growth processes
}

\author{
Subhadip Raychaudhuri ${ }^{(1)}$, Yonathan Shapir ${ }^{(1,2)}$ \\ (1) Department of Physics and Astronomy, University of Rochester, Rochester, NY 14627, USA \\ ${ }^{(2)}$ Department of Chemical Engineering, University of Rochester, Rochester, NY 14627, USA
}

(November 8, 2018)

\begin{abstract}
The scaling properties of the roughness of surfaces grown by two different processes randomly alternating in time, are addressed. The duration of each application of the two primary processes is assumed to be independently drawn from given distribution functions. We analytically address processes in which the two primary processes are linear and extend the conclusions to non-linear processes as well. The growth scaling exponent of the average roughness with the number of applications is found to be determined by the long time tail of the distribution functions. For processes in which both mean application times are finite, the scaling behavior follows that of the corresponding cyclical process in which the uniform application time of each primary process is given by its mean. If the distribution functions decay with a small enough power law for the mean application times to diverge, the growth exponent is found to depend continuously on this power law exponent. In contrast, the roughness exponent does not depend on the timing of the applications. The analytical results are supported by numerical simulations of various pairs of primary processes and with different distribution functions. Self-affine surfaces grown by two randomly alternating processes are common in nature (e.g., due to randomly changing weather conditions) and in man-made devices such as rechargeable batteries.
\end{abstract}

\section{INTRODUCTION}

\section{A. Motivation and background}

The scaling approach to kinetic roughening of surfaces has been found to be very useful in a large number of systems [1-7]. It is based on their self-affine property, reflected by the power-law dependence of the roughness on the system size in the, steady state, large-time regime. In the early regime, the roughness also increases as a power law of the time. Systems in which such a behavior of the surface roughness was observed and analyzed include different (vapor, electrochemical, epitaxial, etc.) deposition processes, burning front, malignant tumors, and others. Similarly, self-affine rough surfaces are formed in opposite processes of recession, desorption, dissolution, etc.

Very recently we have addressed the scaling behavior of cyclical processes in which two different processes (typically one growth and one recession) follow each other regularly. Such cyclical processes are abundant in natural and artificial systems. Many natural growth processes are affected by changing degree of illuminations through the daily cycle or seasonal variations in weather conditions. Rechargeable batteries, charged and discharged periodically, provide an example of a practical application in which a rough metal surface accumulates on one of the electrode (and cause a short when it reaches the other electrode). Chemotherapeutic (or radiation) treatment of a malignant tumor applied periodically and the expansion/curtailment of a bacterial colony depending on the availability of the nutrient, may provide examples in the biosciences. Experiments on the roughness of silver surface grown by cyclical electrodeposition/dissolution were found to be consistent with the scaling predictions. The main results of our theoretical investigations are summarized in Sec. II.

While studying these cyclical processes it was also realized that many of them are not exactly periodic. Namely the duration of one (or both) primary process, may not be uniform. For example, the charge and discharge time of a battery maybe very irregular depending on the way it is utilized. If a growth process depends on atmospheric conditions such as alternating rain and sunshine, the length of the application time of each primary process will also fluctuate drastically. That may be the case for alternating dry/wet erosion processes of a natural rock bed or a man-built wall.

So two crucial questions which ought to be addressed are: To what extent the cyclical theory applies to systems with non-uniform application times of the primary processes? And if the cyclical theory fails to describe them, how are their scaling properties modified? The goal of the present work is to seek and provide the answers to these two essential questions. 


\section{B. Basic scaling concepts and relations}

The roughness of self-affine surfaces created by growth or recession processes have been described using scaling concepts [8]. The width $W(L, t)$ of a surface linear dimension $L$ at a time $t$ is given by

$$
W(L, t)=\left\langle(h(\vec{r}, t)-\langle h(\vec{r}, t)\rangle)^{2}\right\rangle^{\frac{1}{2}},
$$

where the angular bra-ket \langle\rangle denotes average over both lateral sites and different realizations of surface configurations. $W(L, t)$ was found to scale as [8]

$$
W(L, t) \sim L^{\alpha} g(L / \xi(t))
$$

where $\xi(t) \sim t^{1 / z}$ is the lateral correlation length. $g$ is the scaling function which behaves such that for large time $\left(t \gg L^{z}\right) W \sim L^{\alpha}$ ( $\alpha$ is the roughness exponent), while in the early-time regime $\left(t \ll L^{z}\right.$ ), W $\sim t^{\beta}$ (where $\beta=\alpha / z$ is the growth exponent). Different surfaces are classified into universality classes which share the same set of scaling exponents. Some generic universality classes are mentioned in subsection C.

For rough surfaces formed by cyclical processes a suitable scaling law in terms of the number of cycles $n$ was introduced [9] [10]. The width $W(L, n)$ for a system of linear size $L$ obeys the following scaling form:

$$
W(L, t)=L^{\alpha} g_{c}\left(L / \xi_{c}(n)\right)
$$

where $\xi_{c}(n) \sim n^{1 / z}$ is the lateral correlation length and $g_{c}$ is the cyclical scaling function.

For large time $\quad n \gg L^{z}: \quad W \sim L^{\alpha}$,

while for $\quad n \ll L^{z}$ : $\quad W \sim n^{\beta}$,

where $\beta=\alpha / z$ is the growth exponent. Again, different universality classes can be defined depending on the values of the exponents $\alpha$ and $\beta$. In most of the cases studied by us, the scaling exponents of the cyclical process were identical to those of one of the primary processes (the so-called dominating process).

In our previous papers on cyclical growth [9] [10] the durations of the primary processes (in every cycle) were assumed to be uniform. However, in many realistic situations the durations of the primary processes will vary from cycle to cycle.

\section{Growth Models and Universality Classes}

The growth processes fall into different universality classes [2]. All processes within one class share the same exponents and their asymptotic continuum stochastic equations differ at most by irrelevant terms (in the renormalization group (RG) sense). Using the symbolic index $i=1,2, .$. to denote different processes, the ones we consider here follow growth equations of the form:

$$
\frac{\partial h(\vec{r}, t)}{\partial t}=A_{i}\{h\}+\eta_{i}(\vec{r}, t)+v_{i}
$$

where $A_{i}\{h\}$ is a local functional depending on the spatial derivatives of $h(\vec{r}, t)$ and the noise $\eta_{i}(\vec{r}, t)$ reflects the the random fluctuations in the deposition process and satisfies

$$
\left\langle\eta_{i}(\vec{r}, t)\right\rangle=0
$$

and

$$
\left\langle\eta_{i}\left(\vec{r}_{1}, t_{1}\right) \eta_{i}\left(\vec{r}_{2}, t_{2}\right)\right\rangle=2 D_{i} \delta^{\tilde{d}}\left(\vec{r}_{1}-\vec{r}_{2}\right) \delta\left(t_{1}-t_{2}\right)
$$

where $\tilde{d}=d-1$ is the substrate dimension.

Some of the generic growth processes we will consider are:

(i) Linear: Random deposition (RD), Edwards-Wilkinson (EW) [11] and Mullins-Herring (MH) [12] [13] [14].

(ii) Nonlinear: Kardar-Parisi-Zhang (KPZ) [15] and Molecular Beam Epitaxy (MBE) [16] [17]. 


\section{The structure factor $(\mathrm{SF})$}

The structure factor is defined as $S(\vec{q}, t)=\langle h(\vec{q}, t) h(\overrightarrow{-q}, t)\rangle$, where $h(\vec{q}, t)$ is the Fourier transform of the height $h(\vec{r}, t)$. In the theoretical analysis of surface growth it is convenient to work in Fourier space and compute first the $\mathrm{SF}$ rather than the roughness $W$ itself. Also, in some experiments the surface is probed by scattering processes which provide its structure factor.

Dynamic Scaling hypothesis Eq. (2) can be translated to the Fourier space such that [2]

$$
S(\vec{q}, t)=q^{-\tilde{d}-2 \alpha} g\left(q / t^{-1 / z}\right)
$$

Surface width $W$ can be readily calculated from $S(\vec{q}, t)$ using the relation $W^{2}(L, t)=\left(1 / L^{\tilde{d}}\right) \sum_{\vec{q}} S(\vec{q}, t)$.

The organization of the rest of the paper is as follows: In the next section (II) we briefly review the theoretical analysis of cyclical processes and their main results.In Section III Some exact results are derived for the case of two linear primary processes with non-uniform application times. In Section IV various probability distributions of the random application times of the primary processes are considered. Results of numerical simulations for both linear and nonlinear primary processes are presented. Sec. VI contains a summary of our results.

\section{REVIEW OF CYCLICAL GROWTH PROCESSES}

Any cyclical growth process consist of two primary processes. The durations for the first and the second processes are $T_{1}=p T$ and $T_{2}=(1-p) T$, respectively. The period of one cycle is $T=T_{1}+T_{2}$. The cyclic growth equation (equivalent to Eq. (4) of a simple growth/recession) in terms of the basic two processes is

$$
\frac{\partial h}{\partial t}=\left[a_{1} h+\eta_{1}+v_{2}\right] \Theta(p-f(t))+\left[a_{2} h+\eta_{2}+v_{2}\right] \Theta(f(t)-p)
$$

where $f(t)$ is defined as the fractional part of $t / T$ and $\Theta(x)$ is the unit step function.

\section{A. Linear Primary Processes}

When both the primary processes are linear and their durations $\left(T_{1}\right.$ and $\left.T_{1}\right)$ are constants the above Eq. (8) can be solved exactly to yield the SF. These results are is briefly described below and details may be found in our previous papers [9] [10].

For a linear process Langevin equation of the form of Eq. (4) can be easily solved in Fourier space to yield the SF

$$
S(q, t)=\exp \{-2 a(q) t\} S(q, 0)+\frac{D}{a(q)}[1-\exp (-2 a(q) t)]
$$

where $S(q, 0)$ is the SF at $t=0$ which contains the information of the initial roughness. We assume $S(q, 0)$ to be zero, i.e. the growth starts from a flat substrate. This will not affect the asymptotic scaling exponents.

We index the two primary processes by $i=1,2$ respectively and define $\overline{a_{i}}=a_{i}(q) T_{i}$. During the 1 st cycle of the cyclical growth, the structure factor generated by the first primary process (of duration $T_{1}=p T$ ) is assigned as the initial condition for the second primary process. The second process lasts for $T_{2}=(1-p) T$ to yield the structure factor $S_{c}(q, 1)$ of the cyclical process after the first cycle. This is again used as the initial structure factor for the first process in the $2 n d$ cycle. If we keep iterating like this, the SF after $n$ cycles becomes

$$
\begin{aligned}
S_{c}(q, n)= & {\left[\frac{D_{1}}{a_{1}} \exp \left(-2 a_{2} T_{2}\right)\left(1-\exp \left(-2 a_{1} T_{1}\right)\right)\right.} \\
& \left.+\frac{D_{2}}{a_{2}}\left(1-\exp \left(-2 a_{2} T_{2}\right)\right)\right]\left[\frac{1-\exp \left(-2 a_{c} n\right)}{1-\exp \left(-2 a_{c}\right)}\right] .
\end{aligned}
$$

where $\bar{a}_{c}=a_{c} T$ with

$$
a_{c}=\left[a_{1} p+a_{2}(1-p)\right]
$$

In the scaling limit of small $q$, Eq. () for the cyclical SF reduces to 


$$
S_{c}(q, n) \sim \frac{D_{c}}{a_{c}(q)}\left[1-\exp \left(-2 a_{c}(q) T n\right)\right],
$$

where the effective noise strength for the cyclic process is defined as

$$
D_{c}=p D_{1}+(1-p) D_{2} .
$$

In terms of the effective cyclic parameters $a_{c}$ and $D_{c}$, the SF (12) for the cyclical process looks very similar to that of a generic linear growth process (see Eq. (9)) if the time variable is replaced by the number of cycles n. Hence, the dynamic scaling analysis (Eq. (7)) of the structure factor can be used to determine the scaling exponents in the case of a cyclical growth.

In the regime of saturated roughness, after large number of cycles $n \rightarrow \infty$, Eq. (12) yields $S_{c}(q, n) \sim \frac{D_{c}}{a_{c}(q)}$. The roughness exponent of the cyclic process is determined by the $q \rightarrow 0$ divergence of $\frac{1}{a_{c}(q)}$. Since $a_{c}=\left[a_{1} p+a_{2}(1-p)\right] T$ and $a_{i}(q) \sim q^{z_{i}}$, it is the process with smaller $z_{i}$ which dominates the asymptotic cyclical roughness. In the growing phase of the interface roughness, the number of cycles $n$ is multiplied by $a_{c}(q)$ in Eq. (12). The process with smaller $z_{i}$ will again dominate in the $q \rightarrow 0$ asymptotic limit. Therefore, the primary process with the smaller dynamic exponent carries over its scaling exponents to the combined cyclical process.

\section{B. Non-linear primary processes}

We extended our analysis to non-linear processes as well by using an approximate RG approach. In this approach we "set aside" the non-linear terms for the first few RG iterations in which all fluctuations on time scales smaller than the time one full cycle are integrated out. These few initial steps deals only with the linear parts of the two primary processes and result in an effective linear process, as described above. At this stage the non-linear terms are added back to the stochastic equation with their bare couplings multiplied by $p$ (respectively, $1-p$ ) to take into account the fraction of time of their operation. The next iterations of the RG process then may be applied in the usual manner. This approach assumes that the approximation made in the few initial iterations will not change the ultimate fixed-points to which the RG flow take the system. We expect this to be the case if the system location in parameter space is not too close to a separatrix between two basins of attraction.

\section{ANALYTICAL CONSIDERATIONS OF RANDOMLY ALTERNATING PROCESSES}

\section{A. Linear primary processes}

Now we are ready to consider the more general case of nonuniform durations of the two primary processes. The time elapsed during the first and the second processes in the $k^{t h}$ application are denoted by $t_{1}^{(k)}$ and $t_{2}^{(k)}$ respectively. So $t_{1}^{(k)}$ and $t_{2}^{(k)}$ are assumed to be random variables obeying specific probability distribution functions ( $\left.p d f\right)$. We also define two new random variables $\xi_{k}=\exp \left[-2 a_{1}(q) t_{1}^{(k)}\right]$ and $\eta_{k}=\exp \left[-2 a_{2}(q) t_{2}^{(k)}\right]$, in terms of which the structure factor of the cyclic process will be expressed.

The SF, after the first primary process has taken place in the first cycle, turns out to be

$$
S\left(q, T_{1}^{(1)}\right)=\frac{D_{1}}{a_{1}(q)}\left[1-\exp \left(-2 a_{1}(q) t\right)\right]=\frac{D_{1}}{a_{1}(q)}\left(1-\xi_{1}\right),
$$

where $S(q, 0)=0$ is assumed for simplicity. Hence, after one complete cycle the SF becomes

$$
S_{c}(q, 1)=\left(\frac{D_{1}}{a_{1}}\right) \eta_{1}\left(1-\xi_{1}\right)+\left(\frac{D_{2}}{a_{2}}\right)\left(1-\eta_{1}\right),
$$

which can now be used as the initial SF for the first primary process of the next cycle. After the end of the $2^{\text {nd }}$ cycle, the $\mathrm{SF}$ is

$$
S_{c}(q, 2)=\left(\frac{D_{1}}{a_{1}}\right)\left\{\xi_{2} \eta_{1} \eta_{2}\left(1-\xi_{1}\right)+\eta_{2}\left(1-\xi_{2}\right)\right\}+\left(\frac{D_{2}}{a_{2}}\right)\left\{\xi_{2} \eta_{2}\left(1-\eta_{1}\right)+\left(1-\eta_{2}\right)\right\} .
$$

Proceeding in this manner we finally arrive at the general expression of the SF after $n$ cycles 


$$
\begin{aligned}
S_{c}(q, n)= & \left(\frac{D_{1}}{a_{1}}\right)\left\{\sum_{r=1}^{n-1}\left(\prod_{i=r+1}^{n} \xi_{i+1} \prod_{j=r}^{n} \eta_{j}\right)\left(1-\xi_{r}\right)+\eta_{n}\left(1-\xi_{n}\right)\right\} \\
& +\left(\frac{D_{2}}{a_{2}}\right)\left\{\sum_{r=1}^{n-1}\left(\prod_{i=r+1}^{n} \xi_{i} \prod_{j=r+1}^{n} \eta_{j}\right)\left(1-\eta_{r}\right)+\left(1-\eta_{n}\right)\right\}
\end{aligned}
$$

(Note the similarity of this sum of products of random variables to that encountered in 1D diffusion in random environment [18]. For that system a similar expression yields the first passage time and the random terms in the products are the ratios of the transition probabilities in the two directions between neighboring sites). Suppose the duration of the primary processes $t_{1}^{(k)}$ and $t_{2}^{(k)}$ are distributed with the $p d f P\left(t_{1}\right)$ and $P\left(t_{2}\right)$, respectively. We need to take the average with respect to these $P\left(t_{i}\right)$ of the SF in order to obtain the scaling behavior of the roughness. However, the above expression of the SF contains various products of the random variables $\xi$ and $\eta$ and in such situations the average behavior might differ from the typical behavior. Since by definition $\xi_{k}=\exp \left[-2 a_{1}(q) t_{1}^{k}\right]$ and $\eta_{k}=\exp \left[-2 a_{2}(q) t_{2}^{k}\right]$, they always take values less than one. Thus, $\left\langle\xi^{m}\right\rangle\left\langle 1\right.$ and $\left\langle\eta^{m}\right\rangle\langle 1(m=1,2 \ldots)$, i.e. all the moments including the averages remain less than unity, and also $\ln \left\langle e^{-2 a_{i} t_{i}^{k}}\right\rangle<0$. This is valid for any normalizable probability distribution due to the fact that the durations are always positive $\left(t_{i}^{k} \geq 0\right)$. As a result, the $\mathrm{SF}$ is not dominated by the higher products of $\xi_{k}$ and $\eta_{k}$, rather the most dominant contribution in the sum will come from the terms linear in the random variables. In that case, the fluctuation around the average will be small and the typical behavior will be well represented by the average. By taking the average of the Eq. (17)

$$
\begin{aligned}
\left\langle S_{c}(q, n)\right\rangle=\left(\frac{D_{1}}{a_{1}}\right)\left\langle\sum_{r=1}^{n-1}\left(\prod_{i=r+1}^{n} \xi_{i+1} \prod_{j=r}^{n} \eta_{j}\right)\left(1-\xi_{r}\right)+\eta_{n}\left(1-\xi_{n}\right)\right\rangle \\
+\left(\frac{D_{2}}{a_{2}}\right)\left\langle\sum_{r=1}^{n-1}\left(\prod_{i=r+1}^{n} \xi_{i} \prod_{j=r+1}^{n} \eta_{j}\right)\left(1-\eta_{r}\right)+\left(1-\eta_{n}\right)\right\rangle \\
=\left(\frac{D_{1}}{a_{1}}\right)\left\{\sum_{r=1}^{n-1}\left(\prod_{i=r+1}^{n}\langle\xi\rangle \prod_{j=r}^{n}\langle\eta\rangle\right)(1-\langle\xi\rangle)+\langle\eta\rangle(1-\langle\xi\rangle)\right\} \\
+\left(\frac{D_{2}}{a_{2}}\right)\left\{\sum_{r=1}^{n-1}\left(\prod_{i=r+1}^{n}\langle\xi\rangle \prod_{j=r+1}^{n}\langle\eta\rangle\right)(1-\langle\eta\rangle)+(1-\langle\eta\rangle)\right\},
\end{aligned}
$$

where $\langle\xi\rangle=\left\langle\exp \left[-2 a_{1} t_{1}^{i}\right]\right\rangle$ and $\langle\eta\rangle=\left\langle\exp \left[-2 a_{2} t_{2}^{i}\right]\right\rangle$. The above expression (18) of the SF has the form of a geometric series which can be easily summed with the result

$$
\left\langle S_{c}(q, n)\right\rangle=\left\{\left(\frac{D_{1}}{a_{1}}\right)\langle\eta\rangle(1-\langle\xi\rangle)+\left(\frac{D_{2}}{a_{2}}\right)(1-\langle\eta\rangle)\right\}\left[\frac{1-\langle\xi\rangle^{n}\langle\eta\rangle^{n}}{1-\langle\xi\rangle\langle\eta\rangle}\right]
$$

This form of the average SF resembles the SF ( Eq. (10)) for the case of uniform durations of the primary processes. In fact, one can easily arrive at Eq. (10) starting from Eq.(19), once the uniformity of $t_{1}^{k}$ and $t_{2}^{k}$ are assumed, i.e. $P(t)=\delta(t-T)$.

The scaling exponents in this case of random application times may be extracted using the small $q$ divergence (as done in the uniform case also) of the average SF (19), given in terms of $\langle\xi\rangle$ and $\langle\eta\rangle$. The averages of the variables $\xi$ and $\eta$ have been defined as

$$
\begin{aligned}
\langle\xi\rangle & =\int e^{-2 a_{1} t_{1}} P\left(t_{1}\right) \\
& =\hat{P}_{1}\left(s=2 a_{1}\right),
\end{aligned}
$$

and

$$
\begin{aligned}
\langle\eta\rangle & =\int e^{-2 a_{2} t_{2}} P\left(t_{2}\right), \\
& =\hat{P}_{2}\left(s=2 a_{2}\right),
\end{aligned}
$$


where $\hat{P}_{i}(s)$ is the generating function (Laplace transform) of $P_{i}(t)$. Laplace transforms of similar random time intervals were considered by Godréche and Luck [19] in the context of renewal processes. In such stochastic processes events occur at the random epochs of time $t_{1}, t_{2} \ldots$ from some time origin $t=0$. Change of sign of the position of a random walker is one such example. The intervals of time between those events are treated as independent and identically distributed random variables, very similar to the random duration of the primary processes in our case. Depending on whether the mean of the probability distribution $P(t)$ is finite or not, two different types of behavior are expected, as already observed in continuous fractal-time random walk [20] and in the case of renewal processes [19].

(i) Random application times with finite means:

When the mean of the probability distribution is finite, we can write the averages of the random variables $\xi$ and $\eta$ in terms of a series

$$
\langle\xi\rangle=1-2 a_{1}\left\langle t_{1}\right\rangle+\text { higher terms }
$$

and

$$
\langle\eta\rangle=1-2 a_{2}\left\langle t_{2}\right\rangle+\text { higher terms. }
$$

In the scaling limit of $q \rightarrow 0$, i.e. small $a_{i}$, the higher order terms can be neglected with the result

$$
\langle\xi\rangle=1-\nu_{1} q^{z_{1}}\left\langle t_{1}\right\rangle \text { and }\langle\eta\rangle=1-\nu_{2} q^{z_{2}}\left\langle t_{2}\right\rangle .
$$

Again invoking the asymptotic limit $q \rightarrow 0$, the primary process with smaller $z_{i}$ (dynamic exponent) dominates the average SF and hence the scaling behavior of the roughness. Therefore, as long as the probability distribution has a finite mean, the scaling exponents corresponding to the average roughness are identical to those of the uniform duration case, with the average duration being the uniform period. In the next section, this will be shown explicitly for various probability distributions. We will also present results of numerical simulations which shows the validity of our conclusion even if one or both of the primary processes contain nonlinearity.

\section{(ii) Random application times with diverging mean:}

In some specific cases of broad distributions the average of the probability distribution diverges. One such example will be distribution with a fat power law tail $t^{-(\mu+1)}$ with $\mu<1$ (this is similar to the behavior found in continuoustime random walks, giving rise to the so-called fractal time behavior [20]. In this case, $\langle\xi\rangle$ and $\langle\eta\rangle$ in the scaling limit $(q \rightarrow 0)$ are given by [19]

$$
\langle\xi\rangle \simeq 1-\kappa_{1}\left(2 a_{1}\right)^{\mu} \text { and }\langle\eta\rangle \simeq 1-\kappa_{2}\left(2 a_{2}\right)^{\mu} .
$$

By putting $a_{i} \sim q^{z_{i}}$, we obtain

$$
\langle\xi\rangle \simeq 1-\kappa_{1}^{\prime}(q)^{\mu z_{1}} \quad \text { and } \quad\langle\eta\rangle \simeq 1-\kappa_{2}^{\prime}(q)^{\mu z_{2}} .
$$

Hence, the dynamic exponents are modified as $\mu z_{i}$. The process with smaller $z_{i}$ will still dominate and yield the cyclical dynamic exponent $z_{\text {new }}=\mu \min \left\{z_{1}, z_{2}\right\}$. Physically this modification is the consequence of the modified relation between the time and the number of cycles. If we ask what is the total time elapsed after $n$ applications of the two primary processes the answer is as follows: As long as the mean duration of one application is finite (i.e., $\mu>1$ for distributions with an algebraic tail), the total time is proportional to $\mathrm{n}$ times this mean duration. For power-law distributions with $\mu<1$ and diverging mean, the dominating contribution to the total time comes from a finite number of applications, each of them lasting a time of the order of $n^{1 / \mu}$. This also implies that for such systems in which the roughness grows with time as $t^{\beta}$ (where $\beta$ is the growth exponent of the primary process with the smaller $z$ ), it will grow as $n^{\beta / \mu}$ with the number of applications $n$.

After a large number of cycles, once the roughness becomes saturated, the roughness exponent $\alpha$ of the grown self-affine surface should not depend on the random application times of the primary processes. As a result, the exponent $\alpha$ will remain unchanged from the corresponding uniform duration case, which is nothing but the $\alpha$ of the primary process with smaller $z$. This is consistent with our prior observation that the growth exponent $\beta$ is modified as $\beta_{\text {new }}=\beta / \mu$, so that the scaling relation $\frac{\alpha}{\beta_{\text {new }}}=z_{\text {new }}$ remains valid. So for $\mu<1$, the exponent $\beta$ changes continuously depending on the values of $\mu$.

For two primary processes with durations having two different values of $\mu$ of the power law distributions the new dynamic exponent becomes $z_{\text {new }}=\min \left\{\mu_{1} z_{1}, \mu_{2} z_{2}\right\}$, and $\beta=\alpha / z_{\text {new }}$, where $\alpha=\frac{1}{2}\left\{z_{\text {new }}-(d-1)\right\}$. If one of the processes (say for $i=1$ ) has a finite mean while that of the second $(i=2)$ is diverging, the value of the $z_{\text {new }}$ will be: $z_{\text {new }}=\min \left\{z_{1}, \mu_{2} z_{2}\right\}$, with similar expressions for $\alpha$ and $\beta$. 


\section{B. Non-linear primary processes}

\section{(i) Application times with finite mean:}

In this case we expect our approximate RG approach, introduced for periodic processes and described in the previous section, to still be applicable. The few initial RG iterations will coarse grain all time-fluctuations smaller than a typical time of the order of the average time period (the sum of the mean application times of the two primary processes). Although the distributions will always allow remaining fluctuations from cycles longer than the mean, their probability decreases with their length and we do not anticipate them to be relevant to the long time behavior.

(ii) Random application times with diverging mean:

In this case the approximate RG cannot be applied since the dominating effect is contributed from a few extremly long applications, rather than from the accumulation of $\mathrm{n}$ applications. This behavior strongly suggests that each of the primary processes should be renormalized independently form the other and their relative contributions should be compared based on their fully-renormalized scaling behavior (contrary to the case (i) above where the renormalization is carried on after the two processes have been combined to a single effective process).

If that would be the case, the former result $z_{\text {new }}=\min \left\{\mu_{1} z_{1}, \mu_{2} z_{2}\right\}$, will hold in general with the $\mathrm{z}(\mathrm{i})$ of the two primary processes whether they are linear or not. The exponent $\beta_{\text {new }}$ will be given by the larger of $\beta(i) / \mu(i)$. $\alpha$ will simply be that of the dominating process determining $z_{\text {new }}$.

(iii) Random application times with one finite and one diverging mean:

If one of the two processes (say the first one) has a finite mean application time while it diverges for the other (the $2^{\text {nd }}$ ), some special care is required. We can again imagine performing a few initial RG iterations until the average duration of the first process is of order one. The duration of the second process will be rescaled by the same rescaling factor but will continue to be dominated by the few extremly long applications. If we have $z_{2}<z_{1}$ to begin with, then $z_{\text {new }}=\mu_{2} z_{2}$ as before. If, on the other hand we begin with $z_{1}<z_{2}$, then $z_{1}$ is unaffected during the initial $\mathrm{RG}$ iterations and we have to compare the contribution from $\mathrm{n}$ applications of the first process with the few extremly long applications of the second one. Again, we expect the smaller of $z_{1}$ and $\mu_{2} z_{2}$ will dominate and yields $z_{n e w}$ of the combined process. In short these results amount to assigning the value $\mu_{1}=1$ for the finite-mean process and using again the same expressions for $z_{n e w}$ and $\beta_{\text {new }}$ in (ii) above.

\section{NUMERICAL SIMULATIONS OF RANDOMLY ALTERNATING GROWTH WITH DIFFERENT APPLICATION TIME DISTRIBUTIONS}

Below we will consider different probability distributions $P(t)$ (both discrete and continuous), and try to determine the scaling behavior using the SF (19). The variables defined in the previous section will continue to be used. The average duration of the two primary processes will be denoted by $T_{1}$ and $T_{2}$ for all types of probability distributions (as long as the average exists and finite).

We have carried out numerical simulation of various linear and nonlinear discrete growth models to verify our results corresponding to different probability distributions. The system size used in our simulation was varied between 128 to 4096 lattice units. Periodic boundary condition is employed to keep the finite size effects to a minimum. A typical cycle consisted of average deposition $\left(T_{1}\right)$ of 8 layers (average number of particles per site) and the same amount of average desorption $\left(T_{2}\right)$. The maximum number of cycles $\mathrm{n}$ was changed between $512-8192$ to reach the saturated roughness phase. The roughness data was taken for $\sim 1000-6000$ independent runs to average over the realizations of the random deposition noise as well as the probability distributions for the random duration.

\section{A. Uniform Distribution}

The durations of the primary processes are equally probable between two limits $T_{\max }$ and $T_{\min }$. Hence, the probability density is $P(t)=\frac{d t}{T_{\max }-T_{\min }}$. This yields

$$
\begin{gathered}
\langle\xi\rangle=\left\langle\exp \left(-2 a_{1} t_{1}\right)\right\rangle \\
=\frac{1}{T_{\max }-T_{\min }} \int_{T_{\max }}^{T_{\min }} \exp \left(-2 a_{1} t_{1}\right) d t_{1} \\
=\frac{1}{2 a_{1}\left(T_{\max }-T_{\min }\right)}\left[\exp \left(-2 a_{1} T_{\min }\right)-\exp \left(-2 a_{1} T_{\max }\right]\right.
\end{gathered}
$$




$$
\begin{gathered}
=\frac{\exp \left(-a_{1} T_{\max }\right) \exp \left(-a_{1} T_{\min }\right)}{2 a_{1}\left(T_{\max }-T_{\min }\right)}\left[\exp \left(a_{1} T_{\max }\right) \exp \left(-a_{1} T_{\min }\right)-\exp \left(-a_{1} T_{\max }\right) \exp \left(a_{1} T_{\min }\right)\right] \\
=\exp \left(-2 a_{1} T_{1}\right) \frac{\sinh \left\{2 a_{1}\left(\Delta T_{1} / 2\right)\right\}}{\left\{2 a_{1}\left(\Delta T_{1} / 2\right)\right\}},
\end{gathered}
$$

where $T_{1}=\left(T_{\min }+T_{\max }\right) / 2$ is the mean time and $\Delta T_{1}=T_{\max }-T_{\min }$. Similarly,

$$
\langle\eta\rangle=\exp \left(-2 a_{2} T_{2}\right) \frac{\sinh \left\{2 a_{2}\left(\Delta T_{2} / 2\right)\right\}}{\left\{2 a_{2}\left(\Delta T_{2} / 2\right)\right\}}
$$

Clearly, the uniform duration case is recovered when $\Delta T_{i}$ vanishes.

In the scaling limit of small $q$,

$$
\langle\xi\rangle \sim \exp \left(-2 a_{1} T_{1}\right) \quad \text { and } \quad\left\langle\eta>\sim \exp \left(-2 a_{2} T_{2}\right),\right.
$$

since $\frac{\sinh \left\{2 a_{i}\left(\Delta T_{i} / 2\right)\right\}}{\left\{2 a_{i}\left(\Delta T_{i} / 2\right)\right\}} \sim 1$ as $a_{i}=q^{z_{i}} \rightarrow 0$. In this limit, the SF reduces to

$$
S_{c}(q, n) \sim \frac{D_{c}}{a_{c}(q)}\left[1-\exp \left(-2 a_{c}(q) T n\right)\right],
$$

where $T=T_{1}+T_{2}$ is the average duration for one complete cycle with $T_{1}=p T$ and $T_{2}=(1-p) T$. The definitions of $a_{c}$ and $D_{c}$ are same as the uniform duration case. Hence, in terms of the mean durations of the primary processes, the above SF is identical to the SF (10) of uniform duration. Thus the scaling exponents corresponding to the mean cyclical roughness will be identical to the scaling exponents of the primary process with smaller $z$, as observed in the case of constant duration of primary processes.

This may be seen in Fig. 1, where two linear processes namely EW and DT (MH universality) growth models are used to simulate cyclical growth with random durations distributed uniformly between two values. The scaling exponents obtained $(\alpha=0.50 \pm 0.02$ and $\beta=0.25 \pm 0.03)$ are EW exponents. However, the mean roughness is slightly higher (Fig. 2) than the roughness of the corresponding uniform duration case. This is expected as $\frac{\sinh \left\{2 a_{i}\left(\Delta T_{i} / 2\right)\right\}}{\left\{2 a_{i}\left(\Delta T_{i} / 2\right)\right\}}>1$ and hence from eqs. (31) and $(32),\langle\xi\rangle>\exp \left(-2 a_{1} T_{1}\right)$ and $\langle\eta\rangle>\exp \left(-2 a_{2} T_{2}\right)$.

If we include nonlinear processes in our simulations the scaling behavior still remain unchanged from the corresponding uniform duration case. In Fig. 3, the mean roughness is plotted against the number of cycles (for different system sizes) using two nonlinear models, namely, the KK model (KPZ universality) and the LD model (MBE universality). The durations of those primary processes were taken to be uniformly distributed with identical average. The result: $\alpha=0.50 \pm 0.02$ and $\beta=0.32 \pm 0.03$ is consistent with the KPZ values same as obtained in the nonrandom case.

\section{B. Poisson Distribution}

The distribution function is given by $P\left(t_{i}\right)=\frac{\exp \left(-T_{i}\right) T_{i}^{t_{i}}}{t_{i} !}$, with $T_{i}$ being the mean. Averaging over this discrete distribution function

$$
\begin{aligned}
\langle\xi\rangle & =\sum_{t=0}^{\infty} \exp (-2 a t) \frac{\exp \left(-T_{1}\right) T_{1}^{t}}{t !} \\
& =\exp \left\{T_{1}\left(\exp \left(-2 a_{1}\right)-1\right)\right\},
\end{aligned}
$$

where $T_{1}$ is the mean duration for the first process. Similarly, $\langle\eta\rangle=\exp \left(T_{2}\left(\exp \left(-2 a_{2}\right)-1\right)\right.$ with $T_{2}$ being the average duration of the second process.

As before, to extract the scaling exponents small $q$, i.e. small $a_{i}\left(\sim q^{z_{i}}\right)$, limit is taken. In this limit $\langle\xi\rangle \sim \exp \left\{T_{1}(1-\right.$ $\left.\left.2 a_{1}-1\right)\right\}=\exp \left(-2 a_{1} T_{1}\right)$, as obtained in the uniform duration case. Hence the scaling exponents corresponding to the average roughness will be same as the uniform duration case. 


\section{Exponential Distribution}

The distribution function $P\left(t_{i}\right)=\frac{1}{T_{i}} \exp \left(-t_{i} / T_{i}\right)$, where $T_{i}$ is the mean value. This yields

$$
\langle\xi\rangle=\frac{1}{1+2 a_{1} T_{1}} \quad \text { and }\langle\eta\rangle=\frac{1}{1+2 a_{1} T_{2}},
$$

with $T_{1}$ and $T_{2}$ being the average duration of the two primary processes. In the asymptotic scaling limit $q \rightarrow 0$, $\langle\xi\rangle \sim 1-2 a_{1} T_{1}$ and $\langle\eta\rangle \sim 1-2 a_{1} T_{2}$. Since $a_{i} \sim q^{z_{i}}$, the process with smaller $z_{i}$ will dominate the SF (19). Hence, the scaling exponents are once again identical to those of the non-random case.

\section{Power-law Distribution}

If the long time behavior of a primary process has a fat tail, then duration of that process can be taken to be distributed as a power law of time with

$$
\begin{aligned}
P(t) & =\mu t^{-(\mu+1)} \quad t \geq 1 \\
& =0, \quad t<1
\end{aligned}
$$

where $\mu$ is the only parameter of the distribution. Average duration is easily calculated for the above p.d.f.

$$
\langle t\rangle=\int_{1}^{\infty} P(t) d t=\mu \int_{1}^{\infty} t^{-(\mu+1)} d t=\left.\frac{\mu}{1-\mu} t^{(1-\mu)}\right|_{1} ^{\infty} .
$$

There are two possibilities:

(i) For $\mu>1$ : $\langle t\rangle=\frac{\mu}{\mu-1}$. Hence the average and all the higher moments are finite.

(ii) For $\mu \leq 1:\langle t\rangle \rightarrow \infty$, the average diverges.

Below we will consider two possibilities separately.

$$
\text { 1. } \mu>1
$$

In the scaling limit $q \rightarrow 0$, the averages of the random durations $\xi$ and $\eta$ are given by (see eq. (23))

$$
\langle\xi\rangle \simeq 1-2 a_{1} T_{1} \text { and }\langle\eta\rangle \simeq 1-2 a_{2} T_{2} .
$$

From the expression of the SF (19) we can see that the process with smaller $z$ will dominate the average SF and hence the roughness. Hence, the scaling exponents should not change from their corresponding uniform duration case.

We simulated $\mathrm{MH}$ deposition and EW desorption alternately, with their duration distributed with a power law tail $(\mu>1)$. Fig. 4 shows the scaling of the average roughness with number of cycles for different values of $\mu>1$. The exponent $\beta \sim 0.25$ for all those cases, which is the $\beta$ of the dominating EW process. The roughness exponent $\alpha$ should not change from its uniform duration value and we obtain the EW exponent $\alpha(=0.48 \pm 0.05)$ in our simulations.

$$
\text { 2. } \mu<1
$$

In section III we have already analyzed this case of diverging mean duration. The growth exponent $\beta$ is supposed to change continuously depending on the value of the parameter $\mu$. This behavior was observed (Fig. 5) in our simulation of the cyclical process combining $(1+1) \mathrm{d}$ EW and MH model. Random durations of both the primary processes obey power law distributions with identical $\mu$. The roughness exponent $\alpha$ retains its value (Fig. 6) corresponding to uniform application time (i.e. the $\mathrm{EW}$ value).

Presence of nonlinearity in one or both the primary processes did not change the essential scaling behavior from the linear case. We simulated nonlinear KK (KPZ) and LD (MBE) models having durations distributed with power law tails (both $\mu>1$ and $\mu<1$ ). For $\mu>1$, no difference was observed in the scaling exponents from the corresponding uniform duration case. For $\mu<1$, however, the growth exponent $\beta$ increased continuously as the parameter $\mu$ decreased to zero, consistent with the scaling behavior found for the linear primary processes. The roughness exponent $\alpha$ remain unchanged in all those cases.

Therefore, for intermittent applications with a diverging average, the dynamic exponent and the growth exponent start to differ from those of the uniform case whereas the roughness exponent remains the same. 


\section{CONCLUSIONS}

We are now in a position to provide the answers to the two central questions we posed at the beginning of this paper:

1. To what extent the cyclical growth behavior describes also randomly alternating processes? - The scaling behavior of the perfectly periodic growth processes continues to hold for random application times as long as the latter have finite means. Small fluctuations in the application times are thus irrelevant. For linear systems it means that the primary process with the smallest dynamic exponent will dominate. For non-linear processes, the approximate RG procedure devised for cyclical processes may still be applied to extract the asymptotic scaling behavior.

2. When cyclical growth fails to describe randomly alternating processes, what are the their new scaling properties? If at least the distribution of one of the application time decays as a power law $t^{-1-\mu}$ with $\mu<1$ (such that its mean diverges), the effective dynamic exponent is reduced by a multiplicative factor $\mu$. For linear processes it definitely implies that the process with the smallest effective dynamic exponent dominates (the value of $\mu=1$ should be assigned for processes with a finite mean). We claim that the same continues to hold for non-linear processes as well. This claim follows from the fact that a primary process with a diverging mean application time is dominated by a few especially long applications and therefore renormalizes independently from the other primary process. Theoretical studies to further confirm this behavior will be most welcome.

As far as experimental investigations are concerned, they should be straightforward for systems with finite means (e.g., by methods similar to those used to measure the scaling behavior of cyclical growth). It will be more challenging to conduct experimental investigations of surface growth by randomly alternating processes where at least one of them has a diverging mean application time.

\section{ACKNOWLEDGMENTS}

This research was supported by the ONR under grant No. N00014-00-1-0057.

[1] Family F and Vicsek T (ed) 1990 Dynamics of Fractal Surfaces (Cambridge: Cambridge University Press)

[2] Barabasi A L and Stanley H E 1995 Fractal Concepts in Surfaces Growth (Cambridge: Cambridge University Press)

[3] Villain J and Pimpinelli A 1995 Physique de la Croissance Crystalline (Paris: Editions Eyrolles)

[4] Halpin-Healy T and Zhang Y C 1995 Phys. Rep. 254215

[5] Krug J 1997 Adv. in Phys. 46139

[6] Kim D, Park H and Khang B (ed) 1997 Dynamics of Fluctuating Interfaces and Related Phenomena (Singapore: World Scientific)

[7] Meakin P 1998 Fractals, scaling and growth far from equilibrium (Cambridge: Cambridge University Press)

[8] Family F and Vicsek T 1985 J. Phys. A 18 L75

[9] Shapir Y, Raychaudhuri S, Foster D G and Jorne J 2000 Phys. Rev. Lett. 843029

[10] Raychaudhuri S, Shapir Y, Foster D G and Jorne J 2001 Phys. Rev. E. 64051604

[11] Edwards S F and Wilkinson D R 1982 Proc. Roy. Soc. London A 38117

[12] Mullins W W 1957 J. Appl. Phys. 28 333; Herring C 1957 J. Appl. Phys. 21301

[13] Das Sarma S and Tamborenea P 1990 Phys. Rev. Lett. 66325

[14] Wolf D E and Villain J 1990 Europhy. Lett. 13389

[15] Kardar M, Parisi G and Zhang Y C 1986 Phys. Rev. Lett. 56889

[16] Lai Z W and Das Sarma S 1991 Phys. Rev. Lett. 662348

[17] Villain J 1991 J. Phys. (france) 119

[18] Bouchaud J P and Georges A 1990 Phys. Rep. 195127

[19] Godréche C and Luck J M 2000 Preprint cond-mat/0010428

[20] Shlesinger M F 1988 Annu. Rev. Phys. Chem. 39 269-290

FIG. 1. $\ln W$ (roughness) vs $\ln n$ (number of applications) of the $\mathrm{MH} / \mathrm{EW}$ process with finite-mean random application times [Inset: $\ln W_{s}$ (maximal roughness) vs $\ln L$ ]. 
FIG. 2. The roughness $W$ vs number of applications $n$ of the $\mathrm{MH} / \mathrm{EW}$ process with finite-mean randomly alternating durations, compared with the uniform (i.e. cyclical) case (log-log plot).

FIG. 3. $\ln W$ (roughness) vs $\ln n$ (number of applications) of the randomly alternating MBE/KPZ process for different system sizes $L$ [Inset: $\ln W_{s}$ (maximal roughness) vs $\ln L$ ].

FIG. 4. $\ln W$ vs $\ln n$ of the power-law distributed MH/EW randomly alternating process for different values of $\mu>1$.

FIG. 5. $\ln W$ vs $\ln n$ of the power-law distributed MH/EW randomly alternating process for different values of $\mu<1$.

FIG. 6. The roughness $W$ vs number of applications $n$ of the power-law $(\mu=0.75)$ distributed MH/EW process for different system sizes $L$ (log-log plot) [Inset: $\ln W_{s}$ (maximal roughness) vs $\left.\ln L\right]$. 


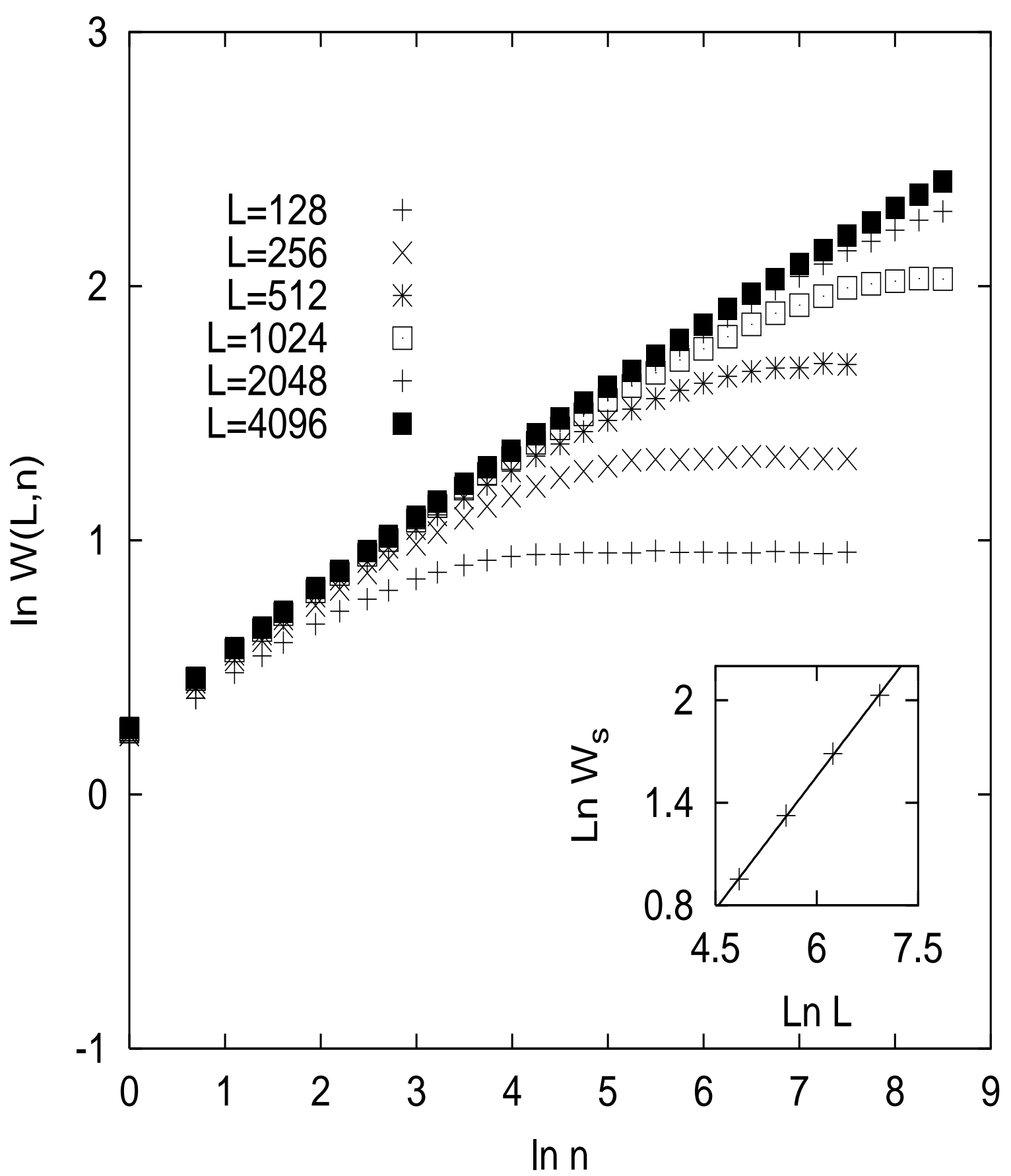

FIG 1 


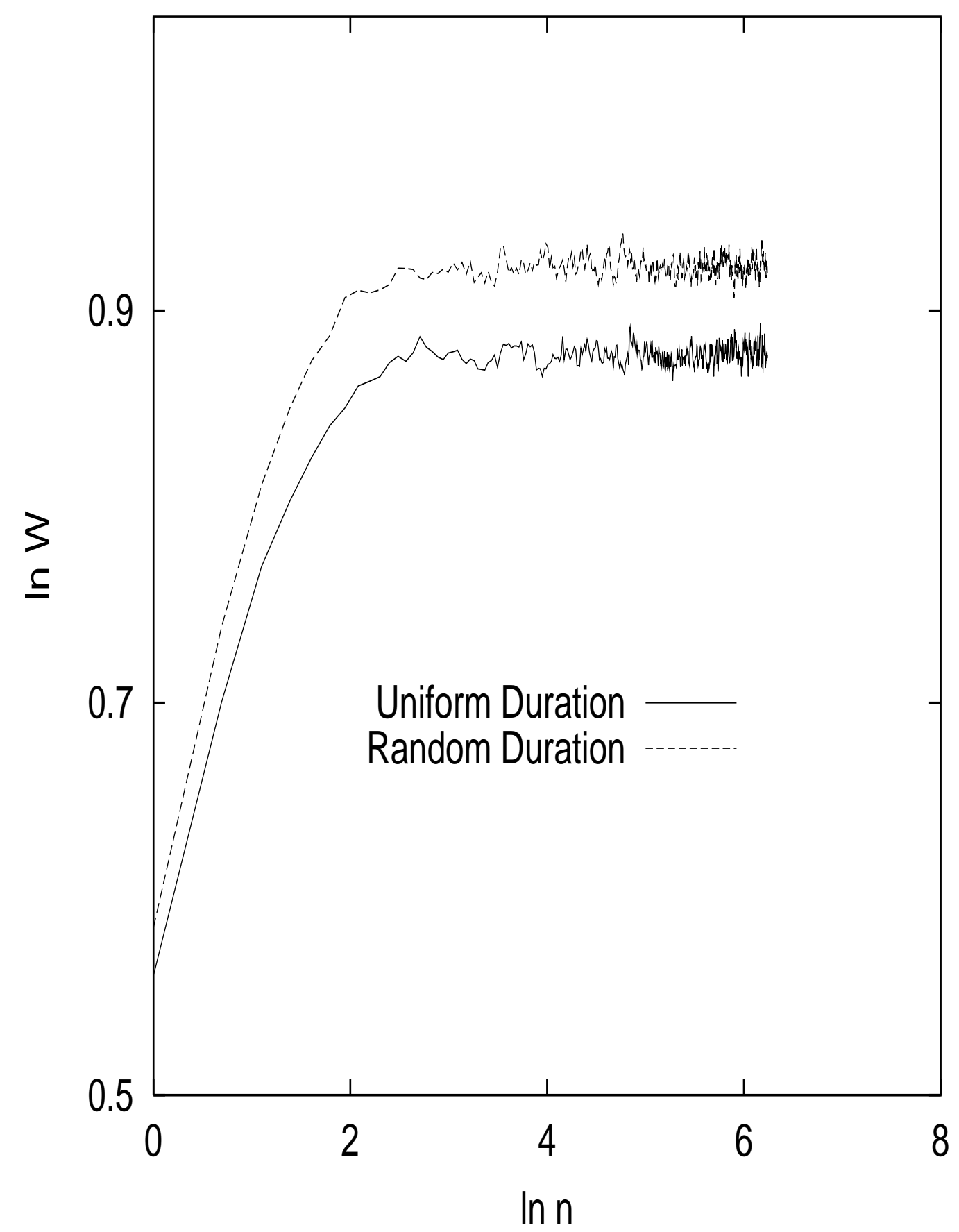

FIG 2 


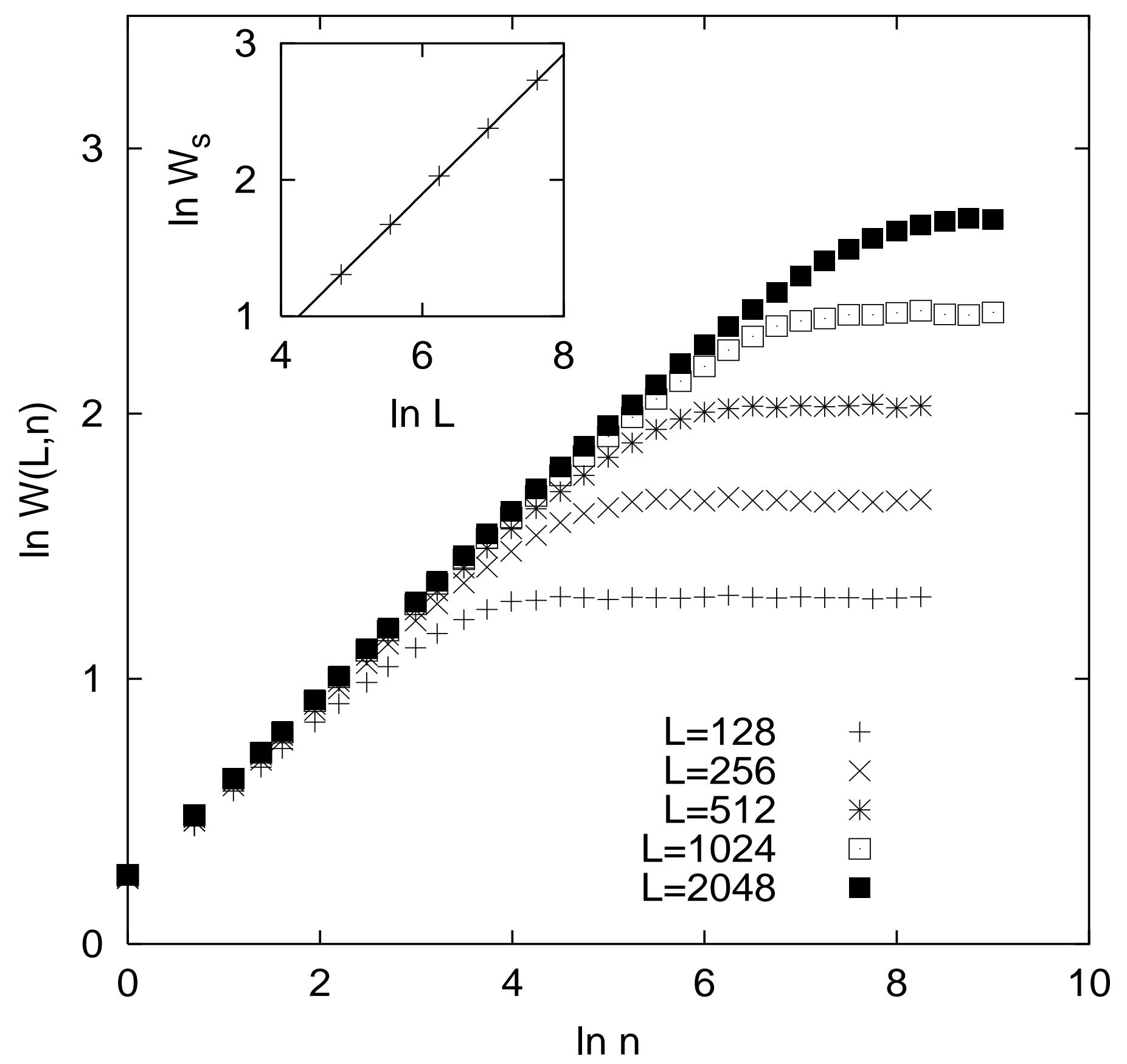

FIG 3 


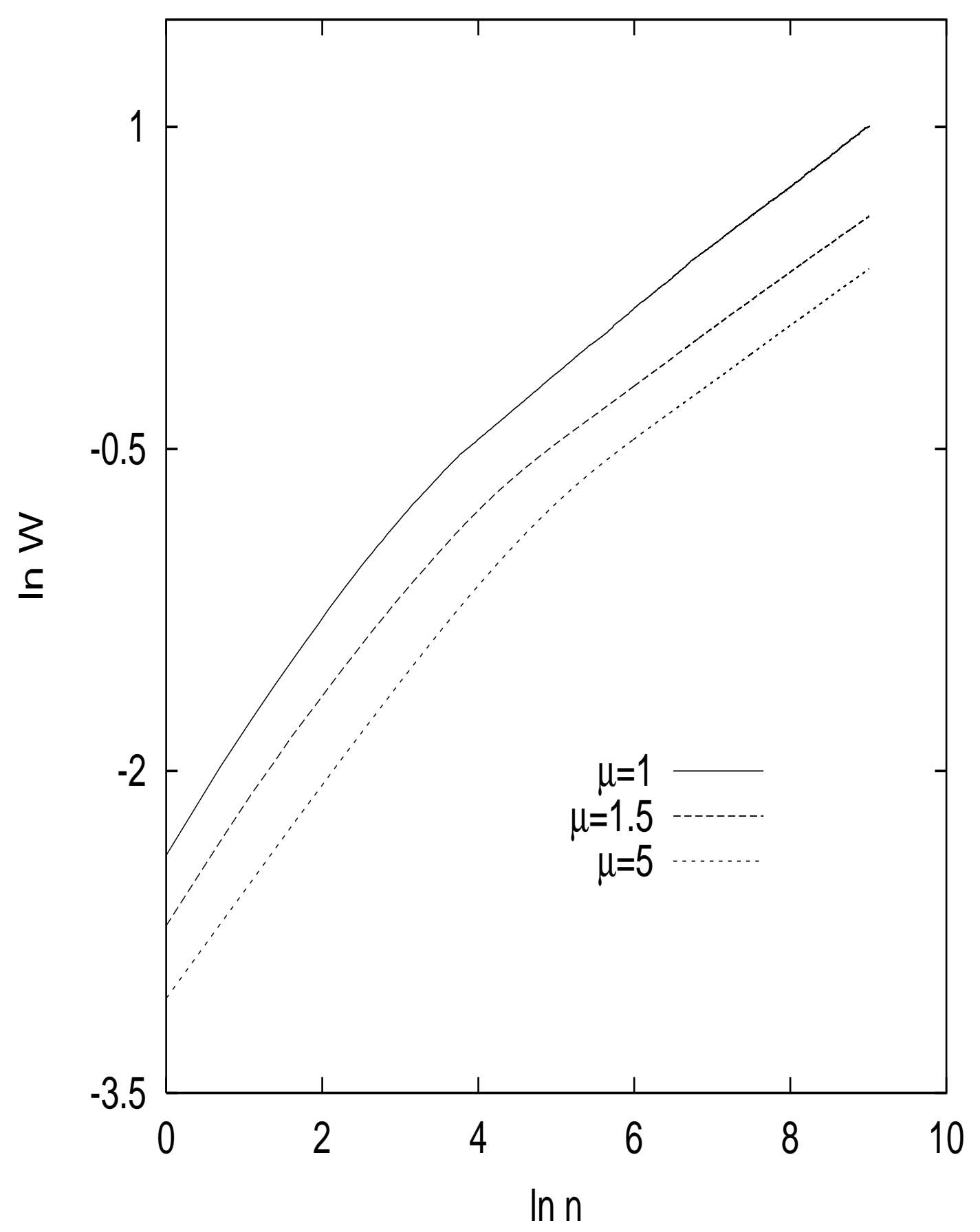

FIG 4 


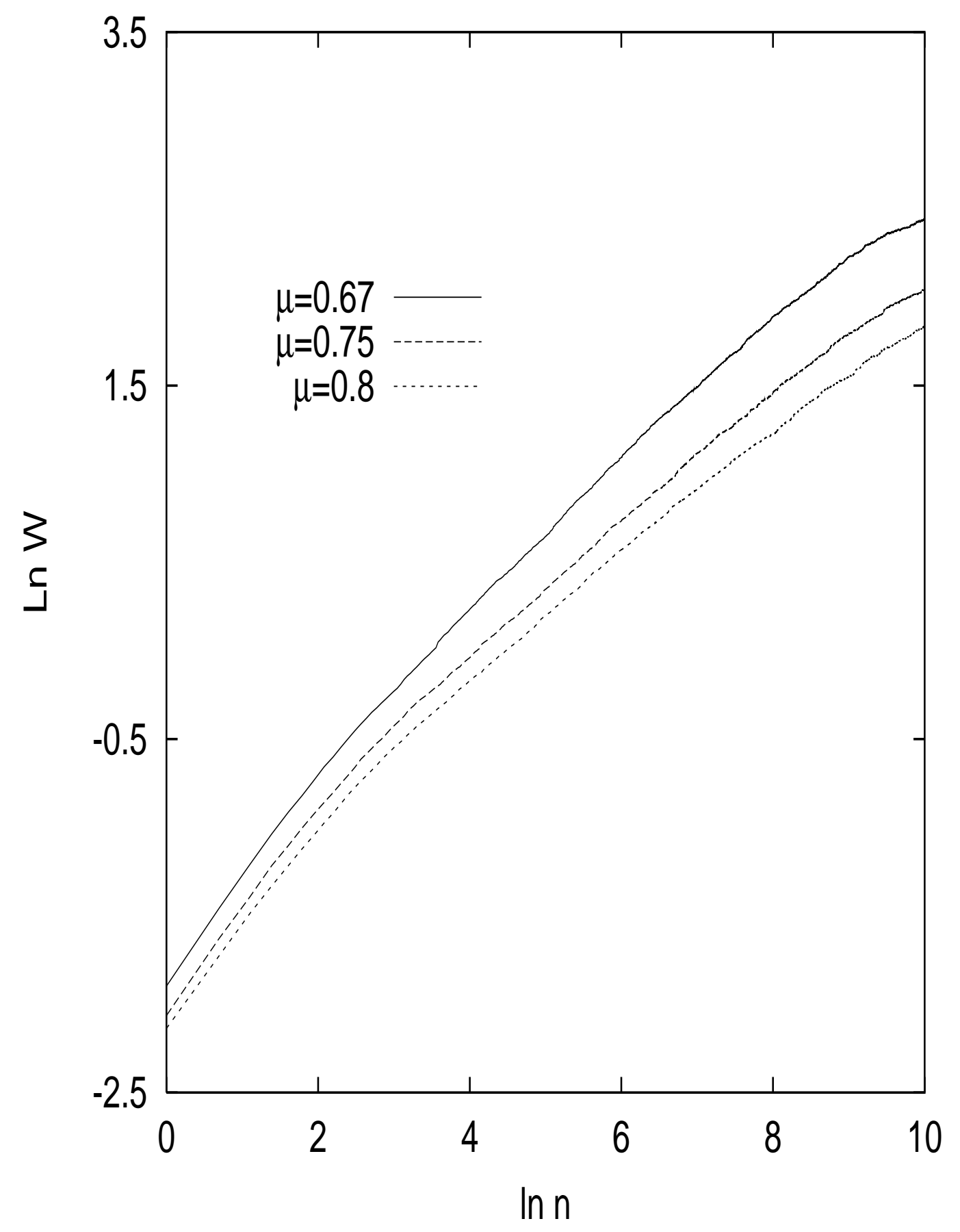

FIG 5 


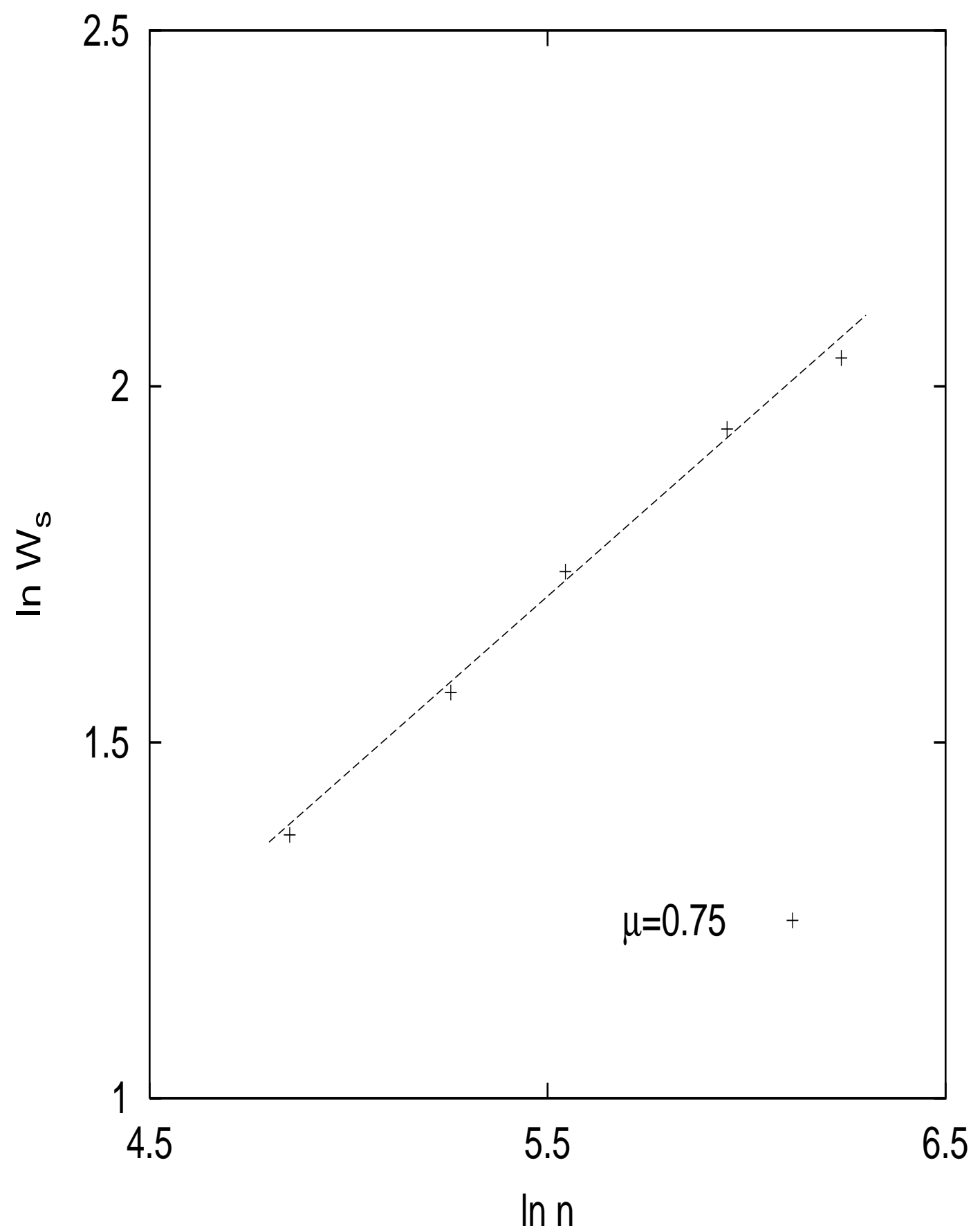

FIG 6 